\title{
THREE PION CORRELATIONS IN SULPHUR-LEAD COLLISIONS AT THE CERN SPS
}

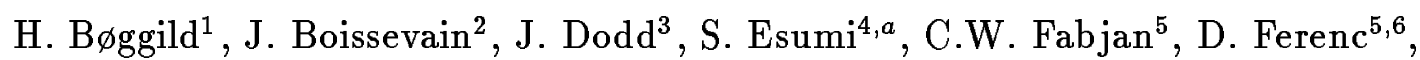
A. Franz ${ }^{7}$, D. Hardtke ${ }^{8, b}$, H. van Hecke ${ }^{2}$ T.J. Humanic ${ }^{8}$, T. Ikemoto ${ }^{4}$, B.V. Jacak ${ }^{9}$, H. Kalechofsky ${ }^{8, c}$, T. Kobayashi ${ }^{10, d}$, R. Kvatadze ${ }^{5, e}$, Y.Y. Lee ${ }^{8}$, M. Leltchouk $^{3}$, B. Lörstad ${ }^{11}$, N. Maeda ${ }^{4, f}$, Y. Miake ${ }^{12}$, A. Miyabayashi ${ }^{11}$, M. Murray ${ }^{13}$, S. Nagamiya ${ }^{3, g}$, S. Nishimura ${ }^{4, h}$, G. Paic ${ }^{5,8}$, S.U. Pandey ${ }^{8, i}$, F. Piuz ${ }^{5}$, V. Polychronakos ${ }^{7}$, M. Potekhin ${ }^{3}$, G. Poulard ${ }^{5}$, D. Rahm ${ }^{7}$, J.M. Rieubland ${ }^{5}$, A. Sakaguchi ${ }^{4, j}$, M. Sarabura ${ }^{2}$, K. Shigaki ${ }^{5, k}$, J. Simon-Gillo ${ }^{2}$, J. Schmidt-Sørensen ${ }^{11}$, W. Sondheim ${ }^{2}$, T. Sugitate ${ }^{4}$, J.P. Sullivan ${ }^{2}$, Y. Sumi ${ }^{4}$, and W.J. Willis ${ }^{3}$

(The NA44 Collaboration)

\begin{abstract}
$\pi^{+} \pi^{+} \pi^{+}$correlations from Sulphur-Lead collisions at $200 \mathrm{GeV} / \mathrm{c}$ per nucleon are presented as measured by the focusing spectrometer of experiment NA44 at CERN. We have investigated the three-pion correlation function at mid-rapidity and found that a genuine three-body correlation is suppressed. A possible interpretation of this result is that the emission of particles is partially coherent.
\end{abstract}

(Accepted for publication in Phys. Lett. B)

1 Niels Bohr Institute, DK-2100 Copenhagen, Denmark.

${ }^{2}$ Los Alamos National Laboratory, Los Alamos, NM 87545, USA.

3 Columbia University, New York, NY 10027, USA.

4 Hiroshima University, Higashi-Hiroshima 739, Japan.

5 CERN, CH-1211 Geneva 23, Switzerland.

${ }^{6}$ Rudjer Boskovic Institute, Zagreb, Croatia.

7 Brookhaven National Laboratory, Upton, NY 11973, USA.

${ }^{8}$ Department of Physics, The Ohio State University, Columbus, OH 43210, USA.

9 State University of New York, Stony Brook, NY 11794, USA.

10 Riken Linac Laboratory, Riken, Saitama 351-01, Japan.

11 University of Lund, S-22362 Lund, Sweden.

12 University of Tsukuba, Tsukuba 305, Japan.

13 Texas A\&M University, College Station, TX 77843-3366, USA.

${ }^{a}$ Now at Universität Heidelberg, D-69120 Heidelberg, Germany.

${ }^{b}$ Now at Lawrence Berkeley National Laboratory, Berkeley, CA 94720.

${ }^{c}$ Now at University of Geneva, Geneva, CH-1211, Switzerland.

${ }^{d}$ Now at University of Tohoku, Sendai 980-77, Japan.

${ }^{e}$ Now at University of Tbilisi State University, Tbilisi, Georgia.

$f$ Now at Florida State University, Tallahassee, FL. USA.

$g$ Now at KEK, Tsukuba 305-0801, Japan.

$h$ Now at University of Tsukuba, Tsukuba 305, Japan.

${ }^{i}$ Now at Wayne State University, Detroit, MI 48202, USA.

${ }^{j}$ Now at Osaka University, Toyonaka-shi, Osaka 560, Japan.

${ }^{k}$ Now at University of Tokyo, Tokyo 188, Japan. 
Particle intensity interferometry provides a method for measuring the space-time extent of a particle-emitting source when the emitted radiation is at least partially incoherent $[1,2,3]$. In particular, two-pion $[4,5,6]$ and two-kaon[7] correlation studies in ultra-relativistic nuclear collisions have provided information on the expansion dynamics and the freeze-out volume when the particles decouple from the source.

Three-particle correlations consist of three combinations of two-particle correlations and a so-called genuine three-body correlation term. Genuine three-body correlations have been observed at high energy for $p \bar{p}$ collisions [8] and $e^{+} e^{-}$-annihilations $[9,10]$. In other reactions and at lower energies, the possible presence of a three-body correlation is unclear $[11,12,13,14]$.

In this paper we measure the genuine three-particle correlation between three pions of the same charge. We parameterize its strength in terms of a weight factor normalized to be unity for a completely chaotic and symmetric system.

Asymmetry of the source will result in a decrease of the strength of the genuine three-body correlation. Source asymmetries might be generated by flow, source geometry and resonance decays, but the effects on the three-body correlation are estimated to be very small[15, 16]. More importantly, coherence of the particle emitting source can also result in a decrease of the strength of the genuine three-body correlation. So far the degree of coherence has been determined from two-particle correlations. This is done by extrapolating the measured correlation function down to zero relative momentum by a simple parameterization, most often a Gaussian. Any deviation from the maximum value of two can be explained as being due to partial coherence. Typically there are several problems encountered at small relative momenta which make a good measurement in that region difficult: shrinking phase space and low statistics, finite momentum and twotrack resolution, and uncertainties in the corrections for the Coulomb repulsion in the case of charged particles. Furthermore, in the case of pion correlations, long-lived resonances such as $\omega, \eta, \eta /$ may give rise to a correlation peak[17] having a width of only a few $\mathrm{MeV}$ on top of the extrapolated Gaussian. This peak is impossible to resolve with current experimental resolutions. Most of these problems are reduced in the case of the genuine three-particle correlation since it is measured over a broad range of relative momenta. The three-particle correlation can therefore provide supplementary information about the reaction mechanism which cannot be determined from the two-particle correlation.

This paper is the first result on the strength of the genuine three-pion correlation in ultra-relativistic nuclear collisions.

\section{EXPERIMENTAL SET-UP}

The layout of the NA44 focusing spectrometer experiment has been described in detail elsewhere[4]. The momentum range is selected by two dipole magnets and is 4 $\mathrm{GeV} / \mathrm{c} \pm 20 \%$ in this analysis. Only one charge sign can be detected in the spectrometer at a time. Three quadrupole magnets create a magnified image of the target in the spectrometer. The data are taken in the "Horizontal" configuration of the quadrupoles, for which the angular coverage of the spectrometer is approximately $-0.3^{\circ}$ to $4.5^{\circ}$ with respect to the incident beam. This setting covers the $p_{T}$ range $0-350 \mathrm{MeV} / \mathrm{c}$ and the rapidity range $3.2-4.1$, with an average $p_{T}$ and rapidity of $121 \mathrm{MeV} / \mathrm{c}$ and 3.8 respectively. The spectrometer uses three highly segmented scintillator hodoscopes for tracking and time-of-flight measurements. The most downstream hodoscope is used for particle identification, and has a time resolution of approximately $100 \mathrm{ps.} \mathrm{A} \mathrm{trigger} \mathrm{is} \mathrm{constructed}$ 
by requiring two or more hits in the hodoscopes. Two threshold Cherenkov detectors provide particle identification at the trigger level, and for the data reported here the trigger additionally requires a pion signal in the first Cherenkov and vetoes electrons in the second Cherenkov. No explicit centrality selection is imposed, however the requirement of at least two particles in the spectrometer favours central events. The trigger is the same as that used in our two-particle analysis $[4,5]$.

\section{THE THEORY OF BOSE-EINSTEIN CORRELATIONS}

The correlation functions for two and three identical particles are given by:

$$
\begin{gathered}
C_{2}=\frac{P\left(k_{1}, k_{2}\right)}{P\left(k_{1}\right) P\left(k_{2}\right)} \\
C_{3}=\frac{P\left(k_{1}, k_{2}, k_{3}\right)}{P\left(k_{1}\right) P\left(k_{2}\right) P\left(k_{3}\right)}
\end{gathered}
$$

where $k_{i}$ is the four-momentum of particle $i, P\left(k_{1}, k_{2}\right)$ and $P\left(k_{1}, k_{2}, k_{3}\right)$ are the two- and three-particle probability densities respectively, and $P\left(k_{i}\right)$ is the single-particle probability density. The probability density can be described for the two-particle case as:

$$
P\left(k_{1}, k_{2}\right)=\iint\left|\Psi_{12}^{B . E} \cdot\left(k_{1} k_{2} ; x_{1} x_{2}\right)\right|^{2} \rho\left(x_{1}\right) \rho\left(x_{2}\right) d^{4} x_{1} d^{4} x_{2}
$$

where $\rho$ is the source density function; here, for clarity, solely a function of $x$. The wavefunction $\Psi$ is defined as the amplitude for a pair produced at $x_{1}$ and $x_{2}$ to register in the detectors, suitably symmetrized for bosons.

For a totally chaotic source the correlation functions for the two- and three-particle cases can be written as (see [3] and references therein):

$$
\begin{gathered}
C_{2}=1+\left|F_{i j}\right|^{2} \quad i j=12,23,31 \\
C_{3}=1+\left|F_{12}\right|^{2}+\left|F_{23}\right|^{2}+\left|F_{31}\right|^{2}+2 \operatorname{Re}\left\{F_{12} F_{23} F_{31}\right\}
\end{gathered}
$$

where, assuming plane wave propagation:

$$
F_{i j} \equiv \int e^{i Q_{i j} x} \rho(x) d^{4} x, \quad Q_{i j}=k_{i}-k_{j}
$$

and where $Q_{i j}$ is the four-momentum difference and $i j$ is the combination of a pair out of the three-particle sample, i.e. $i j=12,23,31$. As seen from Eq. (5), the three-particle correlation function is composed of two parts: the first consists of three terms $F_{i j}$ which we can also determine from the two-particle correlations; the second is the so-called genuine three-particle correlation, in this case expressed as $2 R e\left\{F_{12} F_{23} F_{31}\right\}$.

For a totally chaotic and symmetric source, $F_{i j}$ is real, and we do not get any new information from this last term as $\left|F_{i j}\right|$ can be determined from the two-particle correlation, Eq. (4). In fact, assuming a chaotic Gaussian, symmetric and momentum independent source density function, $\rho(x)$, of width $R$ the correlation functions become:

$$
\begin{gathered}
C_{2}\left(Q_{i j}\right)=1+\lambda e^{-R^{2} Q_{i j}^{2}} \\
C_{3}\left(Q_{12}, Q_{23}, Q_{31}\right)=1+\lambda \Sigma_{i<j} e^{-R^{2} Q_{i j}^{2}}+2 \lambda^{3 / 2} e^{-\frac{1}{2} R^{2}\left(\Sigma_{i<j} Q_{i j}^{2}\right)}
\end{gathered}
$$

where $\lambda$ is a phenomenological parameter[18] equal to the correlation at $Q_{i j}=0$. This parameter has been introduced due to the fact that the measured two- and three-particle 
correlation functions never reach the full value which, for Bose-particles, are 2 and 6 respectively. Note that the $\lambda$ and $R$ in Eqs. (7) and (8) are describing the same system.

Our statistics do not allow a three-dimensional analysis of the three-particle correlation function, Eq. (8). We therefore use a one-dimensional parameterization:

$$
C_{3}\left(Q_{3}\right)=1+\lambda_{3} e^{-R_{3}^{2} Q_{3}^{2}}
$$

where $Q_{3}^{2}=Q_{12}^{2}+Q_{23}^{2}+Q_{31}^{2}$. Eq. (9) is a commonly used parameterization for low-statistics three-particle correlation functions. We will use Eqs. (7) and (9) to parameterize our data.

We introduce a weight factor $\omega$ by the relation:

$$
C_{3}=1+\left|F_{12}\right|^{2}+\left|F_{23}\right|^{2}+\left|F_{31}\right|^{2}+2\left|F_{12}\right|\left|F_{23}\right|\left|F_{31}\right| \times \omega
$$

The weight factor $\omega$ is a measure of the strength of the genuine three-particle correlation. It can be experimentally determined using the following expression, extracted from Eq. (10):

$$
\omega=\frac{\left\{C_{3}\left(Q_{3}\right)-1\right\}-\left\{C_{2}\left(Q_{12}\right)-1\right\}-\left\{C_{2}\left(Q_{23}\right)-1\right\}-\left\{C_{2}\left(Q_{31}\right)-1\right\}}{2 \sqrt{\left\{C_{2}\left(Q_{12}\right)-1\right\}\left\{C_{2}\left(Q_{23}\right)-1\right\}\left\{C_{2}\left(Q_{31}\right)-1\right\}}}
$$

For a totally chaotic and symmetric source $\omega=1$, but $\omega$ will differ from 1 for either an asymmetric or coherent particle emitting source.

In the case of asymmetry, the Fourier transform, Eq. (6), will be complex, $F_{i j}=$ $\left|F_{i j}\right| e^{i \phi_{i j}}$. The phase-factors, $e^{i \phi_{i j}}$, cancel out in the two-particle correlation function, Eq. (4), but survive in the three-particle correlation as seen from the last term in Eq. (5). From Eq. (5), using $\omega=\cos \left(\phi_{12}+\phi_{23}+\phi_{31}\right)$, we get Eq. (11) and $\omega$ can be interpreted as a measure of a phase factor. Asymmetry in the production mechanism will result in a small reduction of $\omega$ from 1 at the level of a few per cent $[16,15]$.

In the case of partial coherence, Eqs. (4-8) are not valid, and more complicated expressions are needed[16, 19]. We can always define $\omega$ by the relation (11) but $\omega$ has a defined meaning as a phase factor only for chaotic sources. If $\omega$ is very different from 1 we can only infer that partial coherence is present; $\omega$ does not measure directly the proportion of pions produced incoherently.

\section{DATA ANALYSIS}

Pions are sampled from an exposure of a Sulphur beam on a Lead target during 1991-92. 24,000 raw events with three tracks are reconstructed from hit positions on the three hodoscopes, with pattern recognition constrained by straight-line trajectories after the magnets. Tracks are not allowed to share the same or neighbouring hodoscope slat to certify a high purity of the three-pion sample. Identical cuts are used in constructing the two- and three-pion data sets. Events containing particles heavier than pions are rejected by the time-of-flight cuts. Contamination of the pion data set by kaons is less than $2 \%$. The $Q_{i j}$ and $Q_{3}$ resolutions are approximately $12 \mathrm{MeV} / \mathrm{c}$ and $16 \mathrm{MeV} / \mathrm{c}$ respectively. These resolutions determine the size of the bins used in this analysis.

The correlation function is determined using $C(\vec{q})=A(\vec{q}) / B(\vec{q})$. The "real" momentum distribution, $A(\vec{q})$, is constructed from tracks from the same event and the "background" distribution, $B(\vec{q})$, is constructed from tracks mixed randomly from all events contained in $A(\vec{q})$. Ideally, the "background" distribution contains all interactions between 
the produced particles, and phase space constraints, but not the Bose-Einstein effect. This method cancels out effects of the experimental acceptance and trigger biases to first order, and is described in our previous publications $[5,7]$.

To compare with theoretical correlation functions, the following corrections[4] are iteratively applied to produce the correlation function $C_{c o r r}$ :

$$
C_{\text {corr }}=C_{\text {raw }} \times K_{S P C} \times K_{\text {acceptance }} \times K_{\text {Coulomb }}
$$

The background spectrum is distorted with respect to the true uncorrelated manyparticle spectrum, owing to the effect of the many-particle correlations on the singleparticle spectrum. This is iteratively corrected by the factor $K_{S P C}$ appropriately generalized to the three-particle case, in which each particle used in the background spectrum is weighted by the correlation from the event it is taken from.

The factor $K_{\text {acceptance }}$ corrects the data for the momentum resolution of the spectrometer and the many-particle acceptance and is calculated by a Monte Carlo programme with a full simulation of the tracking detectors and multiple scattering.

The two-particle correlation function has been corrected for the Coulomb interaction, $K_{\text {Coulomb }}$, between the particles using the Coulomb wave function integration $\operatorname{method}[20,21]$ :

$$
K_{\text {Coulomb }}\left(Q_{12}\right)=\frac{\iint d^{3} x_{1} d^{3} x_{2} \rho\left(x_{1}\right) \rho\left(x_{2}\right)\left|\Psi_{\text {Coul }}\left(Q_{12}, x_{1}-x_{2}\right)\right|^{2}}{\iint d^{3} x_{1} d^{3} x_{2} \rho\left(x_{1}\right) \rho\left(x_{2}\right)\left|\Psi_{\text {planewave }}\left(Q_{12}, x_{1}-x_{2}\right)\right|^{2}}
$$

where $\Psi_{\text {Coul }}\left(Q_{12}, x_{1}-x_{2}\right)$ is the symmetrized non-relativistic two-particle Coulomb wave function, $\Psi_{\text {planewave }}\left(Q_{12}, x_{1}-x_{2}\right)$ is the symmetrized plane wave, and $\rho\left(x_{i}\right)$ is the density distribution of the source. The wave functions are expressed in the two-particle center-ofmass frame, and $\rho\left(x_{i}\right)$ is taken as a Gaussian distribution of width $R$ in all three spatial dimensions.

The three-particle correlation function has been corrected for the Coulomb interaction using a similar technique. A convenient and useful representation of a wave function for the three-particle system with the correct asymptotic wave function is given by $[22,23]$

$$
\Psi_{k_{1} k_{2} k_{3}}\left(x_{1}, x_{2}, x_{3}\right) \sim \psi_{Q_{12}}\left(x_{1}-x_{2}\right) \psi_{Q_{23}}\left(x_{2}-x_{3}\right) \psi_{Q_{31}}\left(x_{3}-x_{1}\right)
$$

Here, $\psi_{Q_{i j}}\left(x_{i}-x_{j}\right)$ is the non-relativistic two-particle Coulomb wave function describing the relative motion of the two particles $i$ and $j$. It is, however, to be emphasized that a wave function of the type Eq. (14) implies that the relative motion of each of the three pairs of particles is independent of that of the other pairs, i.e. that no correlations between the motions of the three particle pairs occur, a notion which clearly can be true at most for asymptotic particle separations.

Given the general three-particle wave function $\Psi_{k_{1} k_{2} k_{3}}\left(x_{1}, x_{2}, x_{3}\right)$ it is straightforward to construct a properly symmetrized wave function for the three-pion system in their center-of-mass system and to calculate the correction factor for the three-particle case with a similar formula to Eq. (13). Details of such a calculation will soon be published[24].

There is still some room for further systematic errors in the Coulomb correction, due to the fact that the unknown exact three-body wave-function is reproduced only asymptotically by the ansatz Eq. (14). However, in the kinematic region of the NA44 experiment, these non-asymptotic corrections are expected to be negligible as the first non-asymptotic correction terms in the three-body Coulomb wave-functions are known 
to decrease strongly with increasing energy of the triplet and the average energy of the triplet is large compared to the typical scale of the three-body Coulomb potential in the NA44 three-pion data sample, see ref. [24] for further details.

Coulomb interactions with the residual nuclear system are negligible but are expected to be small for particles with identical charge-to-mass ratios. Final-state strong interactions are also expected to be small but due to large uncertainties in proposed procedures, no corrections are applied to them[25].

The iterative procedure converges within 3 iterations.

The systematic errors are evaluated by varying the analysis parameters and seeing the difference in the correlation produced. These variations include changing the momentum resolution assumed in the Monte-Carlo correction by $\pm 10 \%$, changing the time-of-flight cuts, and changing the minimum slat separation from one to two slats in all the hodoscopes. The $\omega$ is being re-calculated from Eq. (11) for each new set of correlation functions. The systematic errors are estimated by summing up the differences to the mean-value for each altered setting.

The statistical error on $\omega$ for each bin is: $\sigma_{\text {stat }}(\bar{\omega})=\sigma / \sqrt{N}$, where $\sigma$ is the variance and $N$ is the number of entries. The uncertainty on $\omega$ due to the statistical error on $C_{2}$ and $C_{3}$ is treated as an additional systematic error. This is added in quadruture to the other systematic error and estimated by calculation $\omega$ by using $C_{3} \pm \sigma_{C_{3}, \text { stat }}$ and $C_{2} \pm \sigma_{C_{2}, \text { stat }}$. The statistical error is much smaller than the systematic error.

\section{$5 \quad$ RESULTS AND DISCUSSION}

The three-pion correlation function is shown in Figure 1 and summarized in Table 1. There are no significant data points below $\approx 16 \mathrm{MeV} / \mathrm{c}$ due to the fact that the requirement of at least one slat between each track on each hodoscope depletes this region while increasing the purity of the three-pion sample. The correlation function is fitted using Eq. (9). The two dashed lines show an estimate of the three-pion correlation function when $\omega=0$ (lower dashed line) and $\omega=1$ (upper dashed line). This is done using Eq. (8) with $\lambda$ and $R$ parameters determined from the two-pion correlation function using a Gaussian parameterization. The data points lie within the boundary given by $0<\omega<1$. Although the $\omega=1$ curve is systematically above the data points on Figure 1 , this curve cannot be excluded.

The two-pion correlation extracted from the three-pion data set is shown in Figure 2 and summarized in Table 2. Within errors, the results are consistent with NA44 measurements made using an ordinary two-particle sample of data taken in the same spectrometer setting with identical cuts applied. The $\lambda_{3}$ value from Table 1 is consistent with $3 \lambda_{2}$ which is expected when $\omega$ is close to zero, see Eq. (10). The data are "minimum-bias" in both cases, although central events are favoured in both samples $[4,5]$ due to the requirement of at least two particles in the spectrometer. The effect on the correlation function due to the presence of a third pion is minimal for our data, indicating a small or negligible genuine three-body contribution. It also indicates that the effect of a pion just outside the NA44 acceptance is negligible as assumed in our previous publications $[4,5,6,7]$.

The strength of the genuine three-body correlation, $\omega$, is calculated using Eq. (11). First we determine the correlation functions, $C_{2}$ and $C_{3}$, as described above. Knowing the two- and three-particle correlation functions we can calculate $\omega$ on an event-by-event basis. The correlation values, $C_{2}\left(Q_{12}\right), C_{2}\left(Q_{23}\right), C_{2}\left(Q_{31}\right)$, and $C_{3}\left(Q_{3}\right)$, are obtained by using $Q_{12}$, $Q_{23}, Q_{31}$, and $Q_{3}$ for each event. We use the actual data points of the correlation function, see Figures 1-2, so as not to be biased by some parameterization. As a result we obtain a 


\begin{tabular}{|l|c|c|c|}
\hline System & $\lambda_{3}$ & $R_{3}(\mathrm{fm})$ & $\chi^{2} / \mathrm{N}_{\text {dof }}$ \\
\hline $3 \pi^{+}$ & $1.35 \pm 0.12 \pm 0.09$ & $2.44 \pm 0.16 \pm 0.14$ & $14.4 / 17$ \\
\hline
\end{tabular}

Table 1: Fit results from three-pion analysis. The errors are statistical and systematic respectively.

\begin{tabular}{|l|c|c|c|}
\hline System & $\lambda$ & $R(\mathrm{fm})$ & $\chi^{2} / \mathrm{N}_{\text {dof }}$ \\
\hline $3 \pi^{+} \rightarrow 2 \pi^{+}$ & $0.44 \pm 0.04$ & $4.45 \pm 0.37$ & $17.1 / 20$ \\
\hline $2 \pi^{+}$ & $0.46 \pm 0.04$ & $4.50 \pm 0.31$ & $18.1 / 16$ \\
\hline
\end{tabular}

Table 2: Results from two-pion analysis from the three-particle data set compared to our previous published results [4]. Errors are statistical only.

distribution of $\omega$ for each $Q_{3}$ bin, see Figure 3. Events are taken in the range $16 \leq Q_{3} \leq$ $64 \mathrm{MeV} / \mathrm{c}$, i.e. in the region of the genuine three-body correlation. In order to avoid poles in the denominator of Eq. (11) events are accepted if $Q_{i j} \leq 60 \mathrm{MeV} / \mathrm{c}$. The results are shown in Figure 3. The $\omega$ factor deviates from unity and we see no $Q$-dependence. The weighted-mean is $0.20 \pm 0.02 \pm 0.19$, indicating that a genuine three-body correlation is suppressed.

Our result is, in fact, in agreement with earlier studies of factorial cumulants searching for genuine higher-order correlations[14]. The only possibility known to us for such a small genuine three-body Bose-Einstein correlation is the presence of partially coherent particle emission in these heavy-ion collisions. This implies that hadrons produced in high-energy heavy-ion collisions seem to be emitted from the interaction region in a different way than hadrons produced in simpler and smaller systems.

\section{CONCLUSIONS}

We have investigated the three-pion correlation function and found that the genuine three-body correlation is suppressed. One possible interpretation of this result is that the emission of particles is partially coherent.

\section{ACKNOWLEDGEMENTS}

The NA44 Collaboration wishes to thank Axel Vischer, Henning Heiselberg, Tamas Csörgo, and Ulrich Heinz for valuable discussions, and the staff of the CERN PS-SPS accelerator complex for their excellent work. We thank the technical staff at CERN and the collaborating institutes for their valuable contribution. We are also grateful for the support given by the Science Research Council of Denmark; the Austrian Fond für Förderung der Wissenschaftlichen Forschung through grant P09586; the Japanese Society for the Promotion of Science, and the Ministry of Education, Science and Culture, Japan; the Science Research Council of Sweden; the US Department of Energy; and the National Science Foundation (Nuclear Physics) through grants PHY8906284 and PHY8958491.

\section{References}

[1] R. Hanbury-Brown and R. Q. Twiss, Nature 178 (1956) 1046.

[2] M. Gyulassy, S. K. Kauffmann, and L. W. Wilson, Phys. Rev. C20 (1979) 2267.

[3] B. Lörstad, Int. J. Mod. Phys. A4 (1989) 2861.

[4] H. Beker et al., Phys. Lett. B302 (1993) 510.

[5] H. Bøggild et al., Phys. Lett. B349 (1995) 386. 
[6] H. Beker et al., Phys. Rev. Lett. 74 (1995) 3340.

[7] H. Beker et al., Z. Phys. C64 (1994) 209-217.

[8] N. Neumeister et al., Phys. Lett. B275 (1992) 186.

[9] P. Abreu et al., Phys. Lett. B355 (1995) 415.

[10] K. Ackerstaff et al., Eur. Phys. J. C5 (1998) 239.

[11] N. M. Agababyan et al., Z. Phys. C68 (1995) 229.

[12] T. Åkesson et al., Z. Phys. C36 (1987) 517.

[13] R. Bock et al., Z. Phys. A343 (1992) 293-299.

[14] E. A. de Wolf et al., Phys. Rep. 270 (1996) 1-141.

[15] H. Heiselberg and A. P. Vischer, unpublished, NBI-97-32 (1997).

[16] U. Heinz and Q. Zhang, Phys. Rev. C56 (1997) 426.

[17] T. Csörgö, B. Lörstad, and J. Zimányi, Z. Phys. C71 (1996) 491.

[18] M. Deutschmann et al., CERN/EP/PHYS 78-1 (Jan. 1978).

[19] T. Csörgö et al., Eur. Phys. J. in press, hep-ph/9812422 (1999).

[20] S. Pratt, Phys. Rev. D33 (1986) 72.

[21] M. Biyajima and T. Mizoguchi, preprint SULDP94-9 (1994).

[22] S. P. Merkuriev, Theor. Math. Phys. 32 (1977) 680.

[23] M. Brauner, J. S. Briggs, and H. J. Klar, J. Phys. B22 (1989) 2265.

[24] E. O. Alt, T. Csörgö, B. Lörstad, and J. Schmidt-Sørensen, submitted to Phys. Lett., LUNFD6/(NFFL-7162) (1998), hep-ph/9812474.

[25] D. Boal, C. Gelbke, and B. Jennings, Rev. Mod. Phys. 62 (1990) 553. 


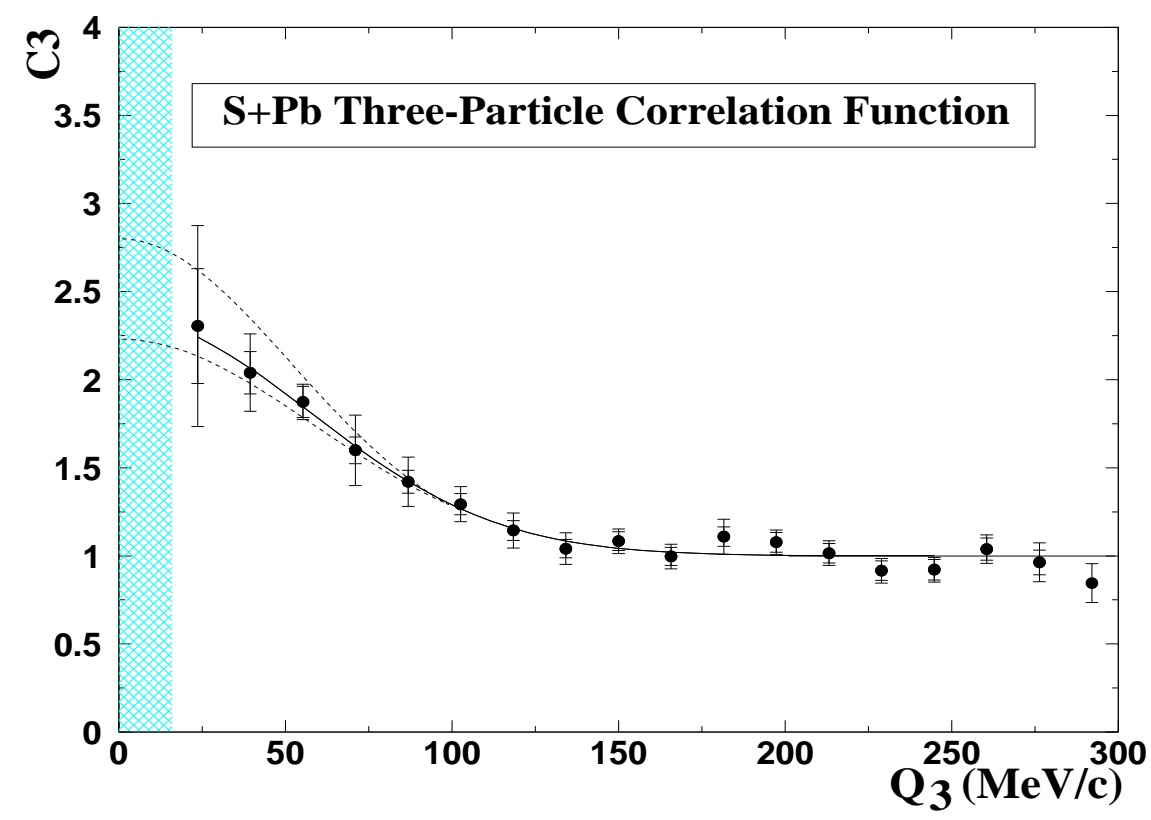

Figure 1: The three-pion correlation function with a fit to Eq. (9) (solid line). The dashed lines are the estimate of the correlation function when $\omega=0$ (lower dashed line) and $\omega=1$ (upper dashed line). The dashed area indicates the $Q_{3}$-range without data. The double error bars are for statistical (inner) and systematic (outer) respectively.

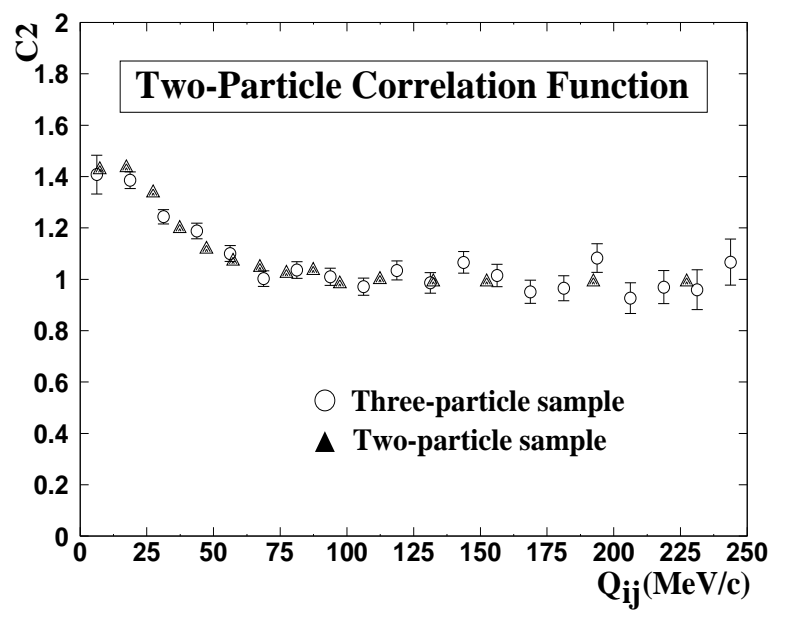

Figure 2: The two-particle correlation function from the three-particle sample compared with the published two-particle sample. Errors are statistical only.

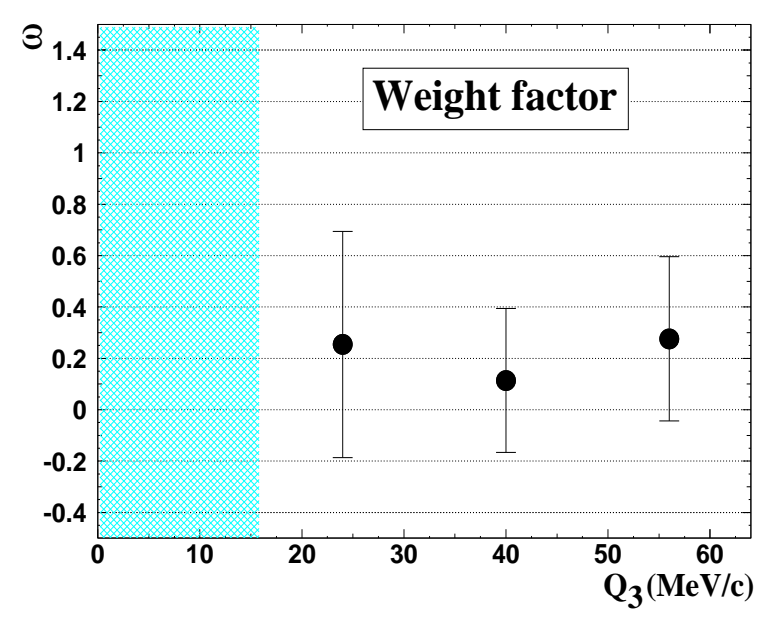

Figure 3: The weight factor $\omega$ as a function of $Q_{3}$. The error bars represent the total error, see the text. 